\title{
Switching dynamics of spatial solitary wave pixels
}

\author{
G. S. McDonald* and W. J. Firth \\ Department of Physics and Applied Physics, John Anderson Building, University of Strathclyde, 107 Rottenrow, \\ Glasgow G4 ONG, UK
}

Received October 2, 1992; revised manuscript received December 29, 1992

Separatrices and scaling laws in the switching dynamics of spatial solitary wave pixels are investigated. We show that the dynamics in the full model are similar to those in the plane-wave limit. Switching features may be indicated and explained by the motion of the (complex) solitary wave amplitude in the phase plane. We report generalization, into the domain of transverse effects, of the pulse area theorem for the switching process and a logarithmic law for the transient dynamics. We also consider, for what is the first time to our knowledge, phase-encoded address of solitary pixels and find that a near-square-wave temporal switching pattern is permitted without (transverse) cross switching.

\section{INTRODUCTION}

Transverse effects in nonlinear optics is a field of both technological and fundamental interest. ${ }^{1}$ The challenge of understanding and controlling spatiotemporal complexity may be reduced when spatially coherent attractors such as solitary waves dominate the transverse patterns. While propagation of solitary wave beams has been realized experimentally with a variety of nonlinear materials, ${ }^{2-4}$ both numerical simulation and analysis predict that spatial solitary waves can also be manifest in the field circulating in nonlinear ring cavities. ${ }^{5}$ In such a configuration they have been proposed as pixels for an optical memory device. ${ }^{6,7}$ Recent experimental realization of binary soliton memory arrays has been achieved in the domain of temporal solitons and with optical fiber loops. ${ }^{8,9}$ Temporal dynamics of bistable devices has already been intensively studied under plane-wave approximations. This paper extends that work and is concerned with the dynamics of spatial solitary wave pixels.

The model is that of an externally pumped passive nonlinear ring resonator in which spatial patterns are restricted to one transverse dimension (Fig. 1). The evolution of the complex field, $G_{n}(x, z)$, during the $n$th circuit of the resonator can be described by ${ }^{5}$

$$
2 i \frac{\partial G_{n}}{\partial z}+\frac{\gamma}{p} \frac{\partial^{2} G_{n}}{\partial x^{2}}+f\left(\left|G_{n}\right|^{2}\right) G_{n}=0,
$$

with the boundary condition

$$
G_{n+1}(x, 0)=A(x)+R \exp \left(i \Phi_{0}\right) G_{n}(x, p) .
$$

The medium nonlinearity is saturable, purely dispersive, and focusing, $f\left(\left|G_{n}\right|^{2}\right)=-1 /\left(1+2\left|G_{n}\right|^{2}\right)$, and beam evolution is parameterized by $p=\alpha_{0} L_{1} / \Delta$, an effective medium length, and $\gamma=\ln 2 /(4 \pi F)$, where $F$ is the (beam) Fresnel number. $\Phi_{0}, \Delta$, and $\alpha_{0}$ are the cavity mistuning, pump detuning, and medium linear absorption coefficient, respectively. $p=2$ and $\Phi_{0}=0.4$ imply a well-developed bistable characteristic and are fixed throughout the simulations. A modulation of the pump field $A(x)$ defines pixel sites on the circulating beam:

$$
A(x)=A_{0}\left(1+M \cos k_{m} x\right) \exp \left(-x^{2}\right) .
$$

For a short period in time ( $T$ cavity transits), narrow address beams are superimposed upon the pump field given by Eq. (3). These beams are spatially Gaussian and temporally square. ${ }^{6,7}$ A spatially uniform array of address beams used to switch solitary waves on a host Gaussian beam implies a range of dynamic and stationary responses over the utilized band of the bistable characteristic. Here we quantify the response of the system during and after address beams and some static characteristics, considering nearly the whole region, in $A$, where there is bistability.

One of the more widely studied transient phenomena in optical bistability is that of critical slowing. ${ }^{10-14}$ Perturbation analysis predicts that when an external parameter $q$ (say, incident intensity or cavity length) crosses its critical value $q_{c}$, then switching time diverges as $\left(q-q_{c}\right)^{-1 / 2} \cdot{ }^{15}$ Such a scaling is widely applicable to many systems that exhibit a first-order phase transition. In our system this slowing effect presents a lower bound on address amplitude for fast switching of a single pixel, while for arrays it creates an upper bound on the hold amplitude (or. alternatively a limitation to packing density ${ }^{16}$ ) when independent switching is to be confidently concluded.

For address pulses a pulse area scaling law was discovered in numerical studies of absorptive bistability. ${ }^{12}$ In Ref. 12 the bias point (background input level) was close to the upswitching threshold. It was found that for a pulse area (temporal duration times amplitude) sufficient to overcome critical slowing, this area alone was the determining factor causing a switch to the upper branch. The law was then extended to a wider class of bistable systems that can be described by simple nonlinear differential equations and whose hysteresis loops are well developed. ${ }^{17,18}$ More recently the subject of noncritical slowing $^{17-21}$ has attracted attention. In this case a slowing in the dynamical evolution occurs far from the (critical) switch points. Analyses were performed that approximate the stationary characteristic to a parabola, and solutions show an approximate logarithmic divergence of lethargy time as pulse width approaches a critical value. ${ }^{21-23}$ In the intermediate region, between the linear scaling of the pulse area theorem and the quadratic scaling of critical slowing, a smooth continuity was found for a wide range of (plane-wave) bistable systems. ${ }^{24}$ Noncritical 


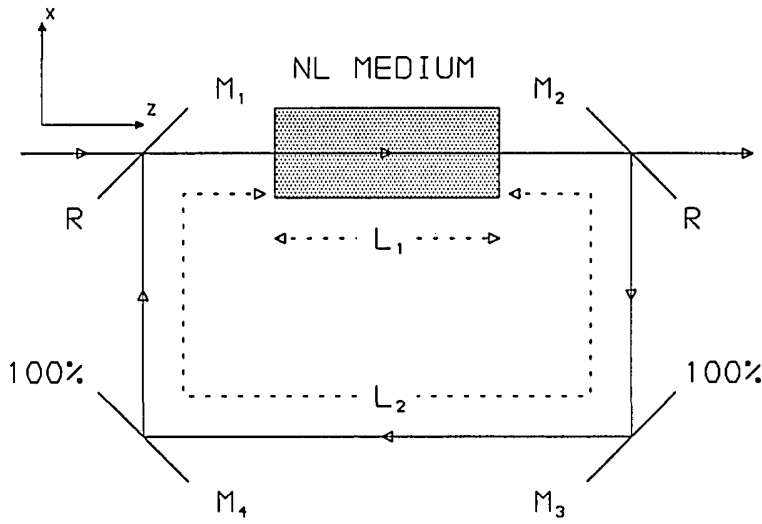

Fig. 1. Schematic of the ring cavity partially filled with a nonlinear medium, $L_{1}=0.3\left(L_{1}+L_{2}\right)$. Mirrors $\mathrm{M}_{1}$ and $\mathrm{M}_{2}$, the input and output couplers, respectively, have a finite intensity reflectivity $R=0.9$.

slowing was a natural candidate for quantitative examination in our system, since it was seen to have a strong effect on switching times even while relatively crude parameter scans in address pulse amplitude were performed.

It is generally thought that fixed scaling laws may dominate the dynamics of optical bistability and transcend the particular physics of any one system. In purely absorptive bistability the phase of the complex field remains constant, removing a degree of freedom that is present in a dispersive system. In this sense we expect less straightforward dynamical scaling dependencies on the fixed points that cannot be considered to be constrained to the real axis. In our system accommodation of transverse effects is an additional issue. We have already demonstrated independent operation of many solitary pixels ${ }^{6,7}$ and thus concentrate here mainly on the study of the dynamics of a single transverse solitary wave pixel.

\section{STATIC AND DYNAMIC SEPARATRICES}

The use of temporally square address pulses provides a simple and definitive, local and overall switch character, whereas local hold beam amplitude $A$ gives a clear indication of bias point. Previous plane-wave studies centered on critical pulse duration $T^{*}$, a parameter that in mapping terms is discrete and experimentally may be ambiguous and difficult to measure. Continuity throughout the whole possible working range of $A$ is a necessary consideration in determination of the applicability of any scaling law. In addition to switch size $A_{S}$ we choose the output solitary pixel amplitude to parameterize our system. This is done in the belief that self-focusing contains the transient spatial structures, during the switching process, in a consistent manner. It is in this sense that we use the term solitary wave in the context of transient solitary amplitudes. We do so in hindsight and shall make further justification of its usage. Certain nominal system parameters will remain fixed and, unless otherwise stated, results pertain to $F=800, T=5, M=0.05$, and $k_{m}=67.32 .^{7}$

To establish the origin of much of the slowing observed in the dynamics of our model, the solitary amplitude during noncritical slowing has been compared with the upperand lower-branch outputs for a range of working points.
In Fig. 2a the three sets of output amplitude are plotted (as points) against $A$, and also each data set has a best-fit curve. This indentifies the static separatrix as the median branch. For the same numerical experiments the switch amplitudes are plotted in Fig. 2b. A switching pulse creates a time-dependent process for which the static separatrix is not a convenient representation and where such a dynamic separatrix needs to be determined. This latter characteristic depends strongly on our address parameters, though the choice of the simplest (square) address shape removes the need to consider some further dependencies.

\section{PLANE-WAVE LIMIT}

For insight into the complexity of switching dynamics in a dispersive device we digress in this section to examine the more tractable and computationally accessible problem of our system in the plane-wave limit. The cavity boundary condition relating the complex intracavity field $g_{n}$ on consecutive transits is in this case ${ }^{5}$

$$
\begin{aligned}
g_{n+1} & =a+R \exp \left\{i\left[\Phi_{0}+N\left(g_{n} g_{n}{ }^{*}\right)(p / 2)\right]\right\} g_{n} \\
& =a+R \exp (i \Gamma) g_{n} .
\end{aligned}
$$
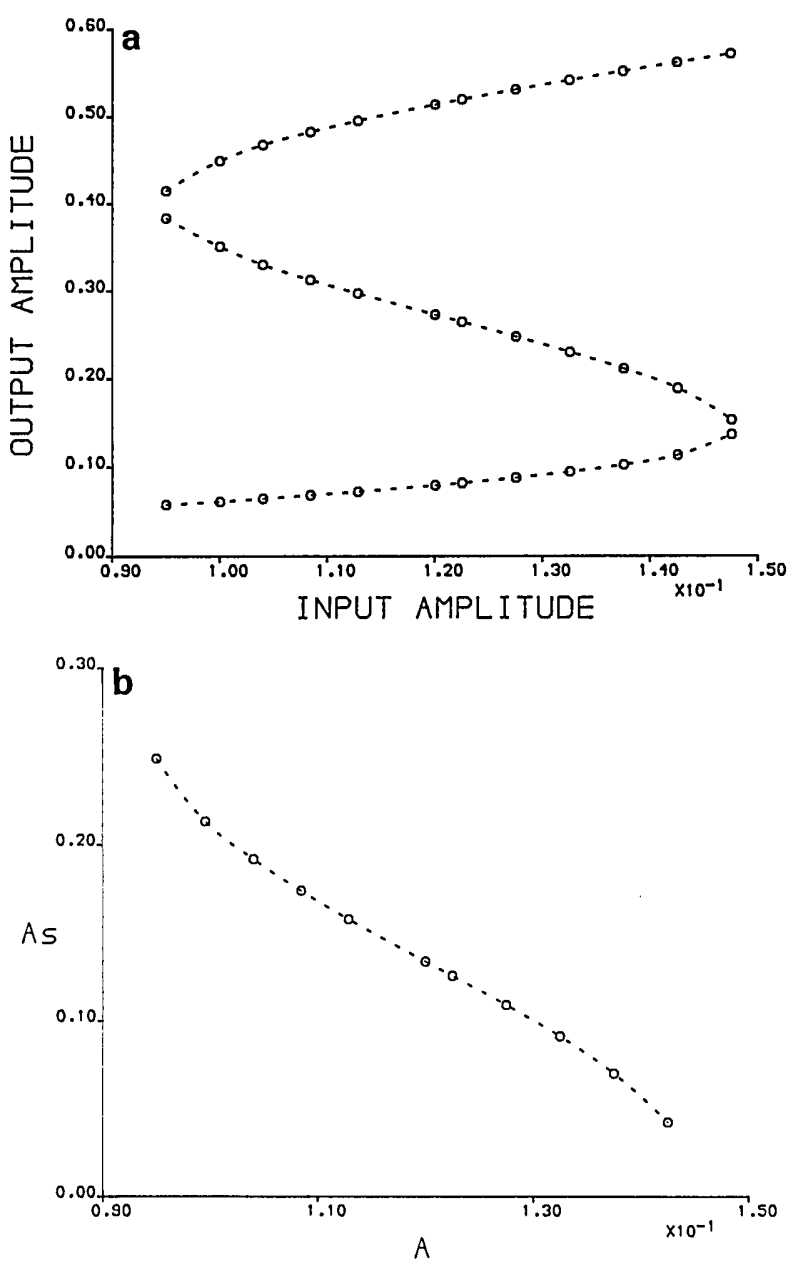

Fig. 2. Separatrices in terms of electric field amplitudes. a, The static separatrix. Output amplitudes when noncritical slowing occurs is compared with those of the lower- and upperbranch fixed points. b, A dynamic separatrix. Variation across the bias range of the required switch amplitude $A_{S}$ for noncritical slowing. 

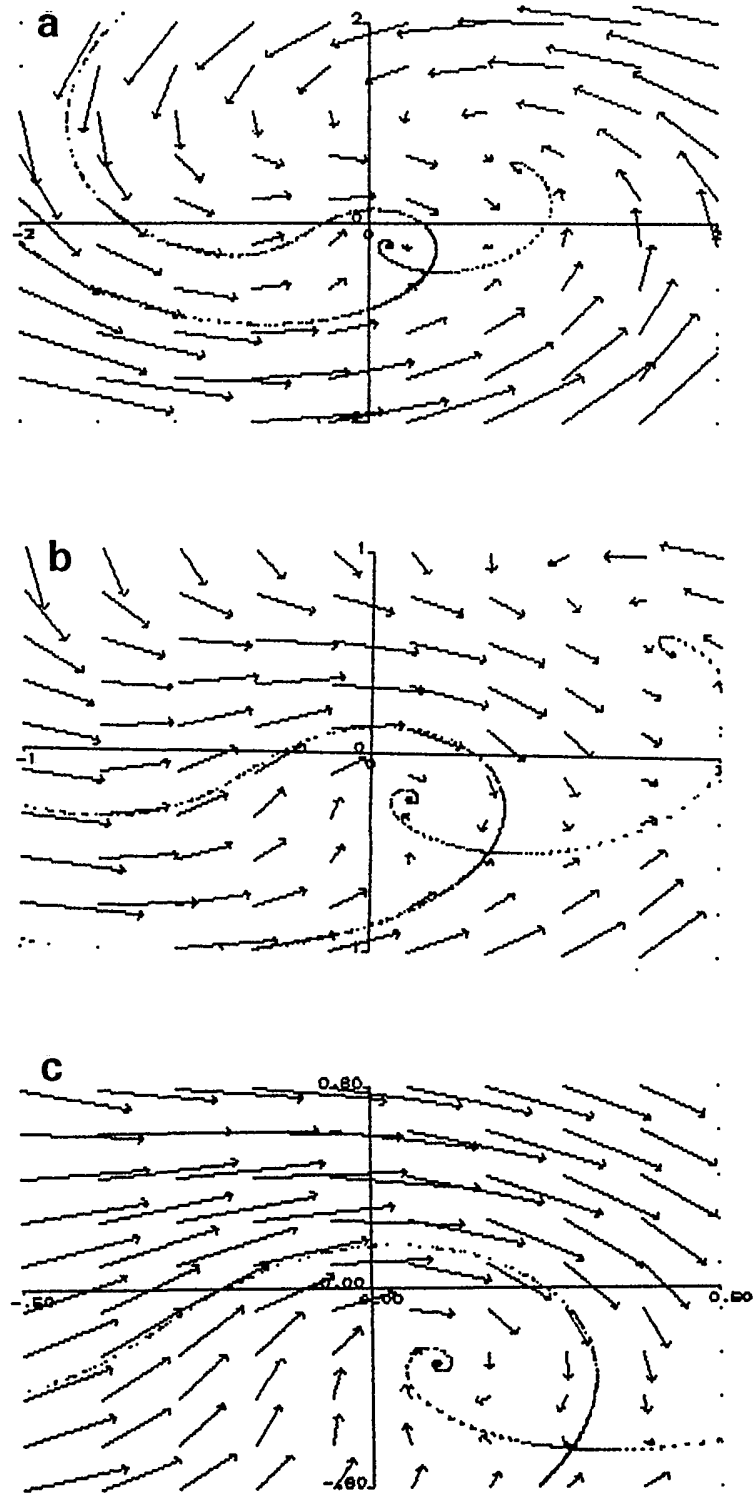

Fig. 3. Samples of the dynamic flow (arrows) and the stable and unstable manifolds (dotted curves) in the plane of the complex electric field. $a,-2 \leq \operatorname{Re}[g] \leq 2 ; b,-1 \leq \operatorname{Re}[g] \leq 1$; c, $-0.5 \leq \operatorname{Re}[g] \leq 0.5$.

The input level is denoted $a$, and we define $\Gamma$ to be the sum of the linear and the nonlinear cavity mistunings. Our reference plane, here, is just after the input mirror, $M_{1}$. Linearizing this map around the fixed point $g$, one finds that the calculated eigenvalues on the upper and the lower branches are conjugate pairs, implying a spiraling dynamic trajectory (stable spiral nodes). ${ }^{25,26}$ However, each point of the median branch is a saddle point. In the neighborhood of each saddle point the stable $\left(W^{s}\right)$ and unstable $\left(W^{u}\right)$ manifolds intersect. These manifolds extend out into the (complex field) phase plane and are fundamental to the transient dynamics. $W^{s}$ and $W^{u}$ have been numerically generated for a range of input amplitudes for which bistability exists. Calculation of $W^{u}$ is relatively straightforward; one considers a cluster of initial conditions around the saddle point. For $W^{s}$ one recognizes that when time is reversed this becomes the unstable manifold, and thus one can use the inverse map. ${ }^{25,26}$ Results for $a=0.12$ are shown as dotted curves in Fig. 3. Since the forward map is dissipative and the dynamic flow is tangential to these manifolds, one can deduce that dynamic trajectories will never cross these lines if the lower- and the upper-branch fixed points ( $L$,and $U$, respectively) are stable. ${ }^{25}$ Thus all initial conditions in the complex plane lying within the lobe of the $W^{s}$ spiral remain within this lobe and ultimately end up at $L$ (switch off). Conversely, initial conditions outside the lobe are attracted to $U$ and thus effect a switch to the on state (the upper branch).

As the median point $M$ approaches $L$ in the complex plane (increasing $a$ and moving closer to the upswitching point), the set of initial conditions that lead to an off state shrink, while as $\mathrm{M}$ approaches $\mathrm{U}$ the $W^{s}$ lobe opens and a spiral arm sweeps around to consume the plane. We are now in a position to appreciate that, when one is considering dispersive bistability, it is not sufficient to have knowledge only of the static separatrix such as is shown in Fig. 2a. This curve is merely a projection of the complete picture. For each value of input amplitude it is the curves $W^{s}$ and $W^{u}$ that actually define whether switching takes place. While these manifolds are instructive, they are asymptotic in nature and do not yield much detail concerning transiency. We may probe deeper into the dynamical effects through consideration of further sets of initial conditions. For each considered window on the complex plane a uniform grid of starting values was defined and used for a single iteration of the map. The results provide a set of dynamic vectors in the plane (direction and amplitude) that furnishes a discrete sampling of the full dynamic flow. These dynamic vectors are also shown in Fig. 3 and not only verify the static nature of $W^{s}$ and $W^{u}$ but also convey information on switching speed. In addition, such figures can explain observed dynamical effects, which are otherwise anomalous, since they account for both the amplitude and phase of the intracavity field.

\section{THE SWITCHING PROCESS}

In the context of a bistable system, switching is, most simply, changing input amplitude sufficiently to create an initial condition far from the (sole) stable equilibrium point. Dynamic evolution in the switching of solitary pixels from the lower branch is shown for a wide range of address amplitudes in Fig. 4. The first 30 transits correspond to bias beam initialization, after which the pixel site at the center of the beam is addressed with a pulse with a duration of 5 transits. $^{7}$ Many such parameter sweeps have been performed, covering the range of $A$ for which it may be feasible to operate a bistable device $(A \simeq 0.095$ " 0.1425$)$. Even with such an abrupt address, noncritical slowing may occur over a time scale of many hundreds of cavity transits (metastable trajectories have been seen to persist for longer than 600 transits). What may, initially, seem surprising is that just after the address pulse there exist small relaxation oscillations around the median solution. Oscillation is more pronounced at lower bias points, where larger address beams are used, and is a transverse effect. A (spatially) local injection raises the peak amplitude, but the pixel subsequently expands to the solitary width, leading to relaxation oscillations. As one can see, this transverse variation is transient and small. In addition to the demonstration of significant noncritical slowing in the switching of solitary pixels, Fig. 4 indicates that simple 
parameterization of the switching process by using only the solitary amplitude may be possible.

Concentrating on the transient response during the switch, we seek a scaling with respect to $A_{S}$ for each value of $A$. To a reasonable approximation, we treat the temporal response as linear. ${ }^{20}$ Linear-fit gradient as a function of $A_{S}$ for three representative bias points is shown in Fig. 5. Response is mostly linear with $A_{S}$ and does not vary greatly over the utilized band in $A$. During a large switch the topology is that of a distant fixed point $U$. The energy balance associated with this fixed point is greatly upset, and regions of temporal linearity reflect local uniformity of the dynamic flow sufficiently far from $U$. Considering curves 1 to 3 , we are moving the bias point along the lower branch and toward the critical point. The cluster of data points on each curve acted as a reference as

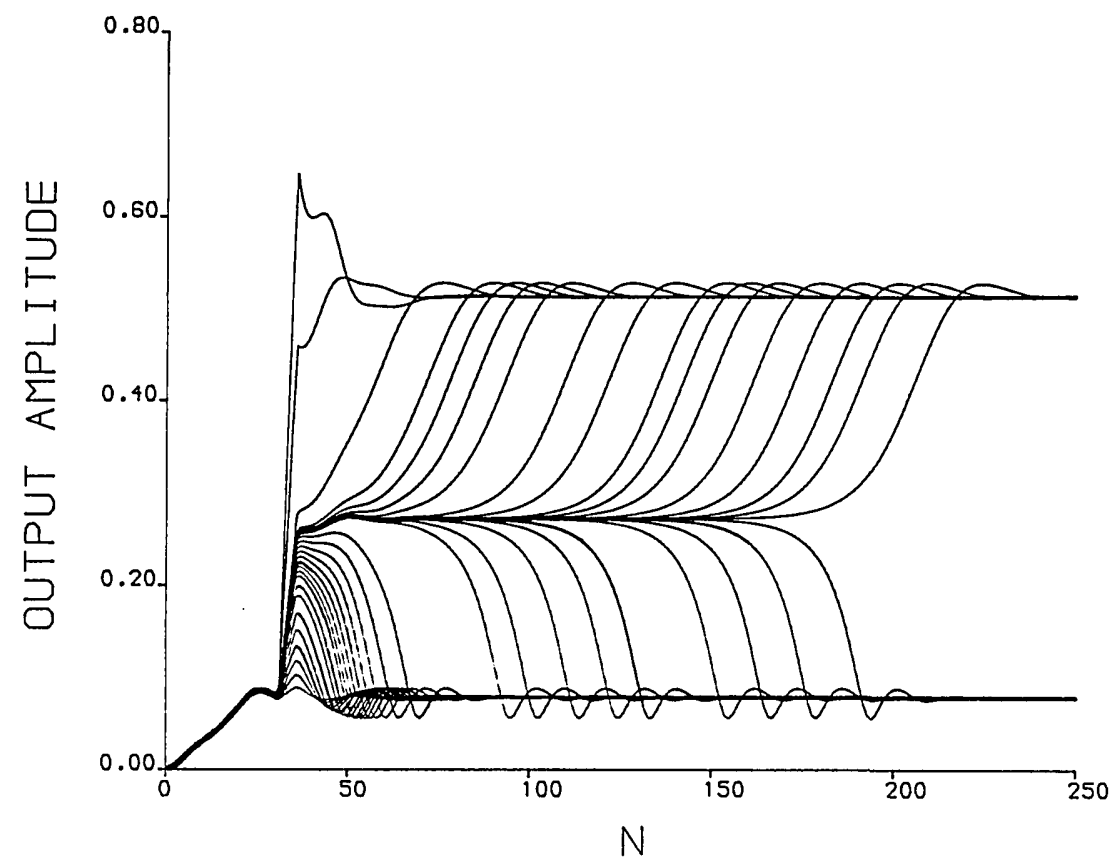

Fig. 4. Temporal evolution of peak output amplitude at $A=0.12$ for a selection of switch pulse amplitudes: $A_{S}=0.0131$ to 0.393 , $N$ is the number of cavity transits.

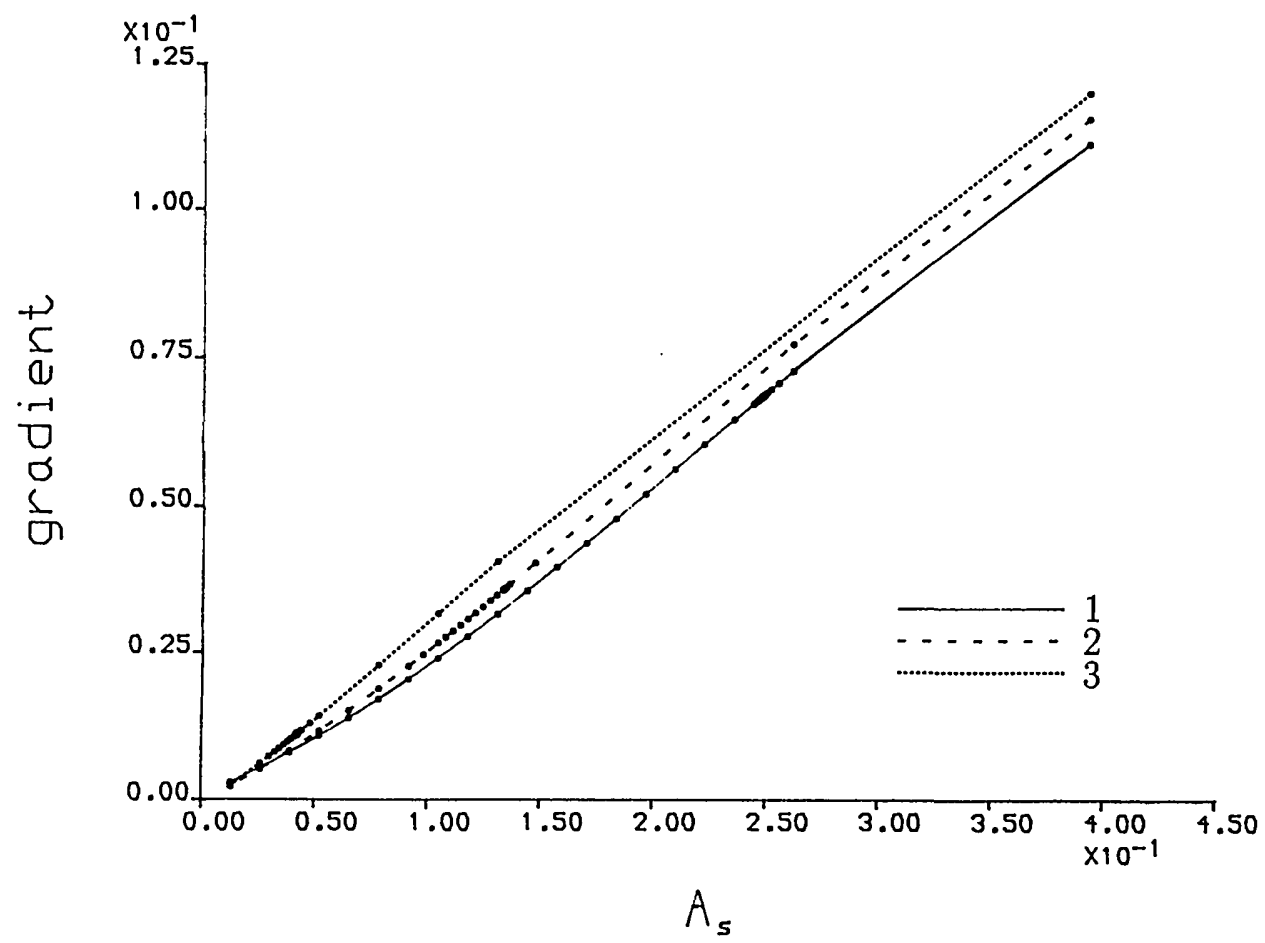

Fig. 5. Rate of increase of the peak output amplitude (with respect to $N$ ) during a switch of amplitude $A_{S}$. Data for three representative bias levels are marked with dots, while the best-fit curve type denotes different values of $A$. Curves 1,2 , and 3 represent $A=0.095,0.12$, and 0.1425 , respectively. 

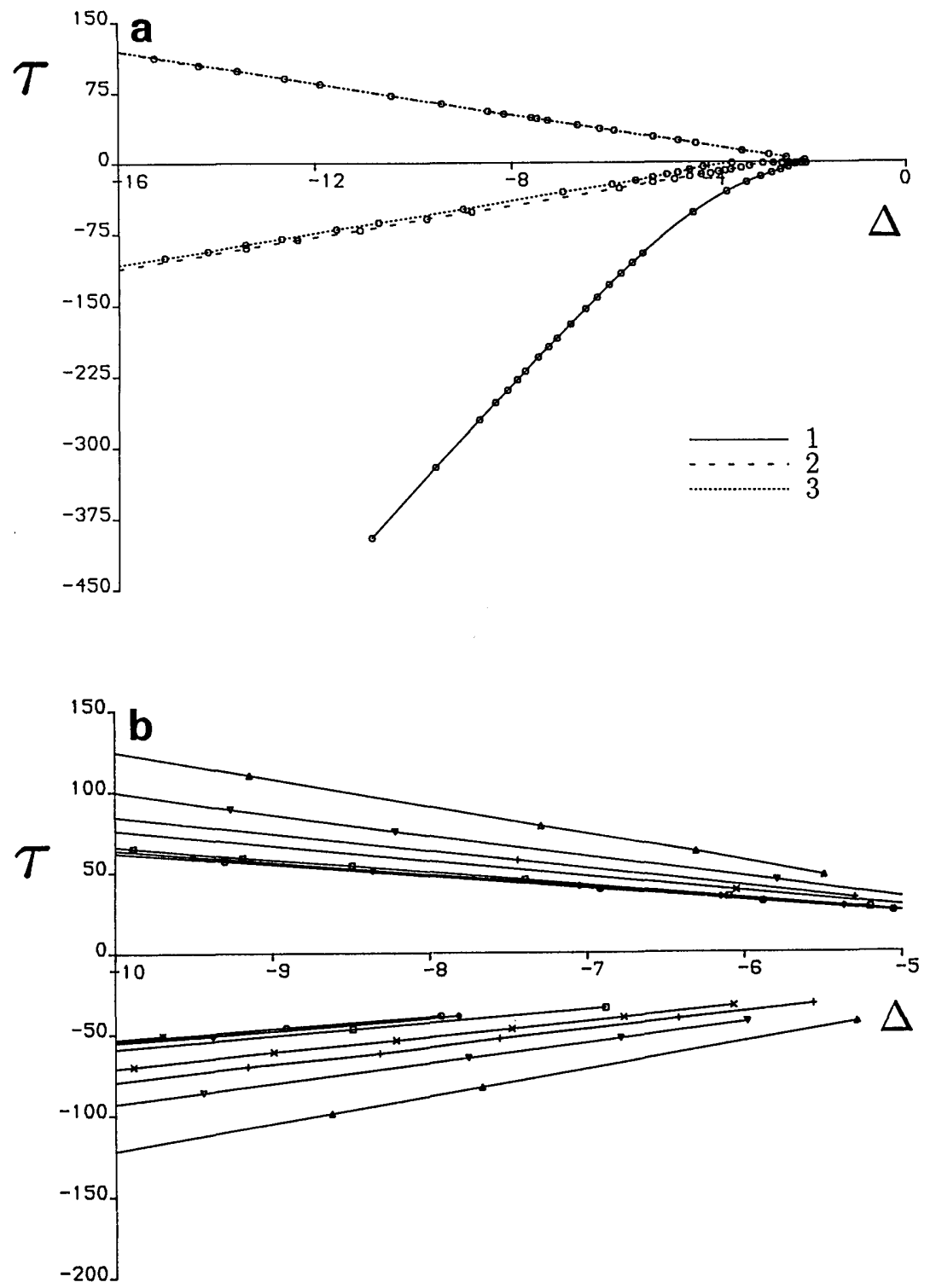

Fig. 6. Dependency of lethargy time, $\tau$, on the difference between switch amplitude $A_{S}$ and its critical value $A_{S}{ }^{*}$, where $\Delta=\ln \mid A_{s}-A_{S} *$. a, Curves 1,2 , and 3 represent $A=0.095,0.12$, and 0.1425 , respectively. b, A range of curves representing $A=0.096$ « 0.1375 .

more simulations were performed for values of $A_{S}$ that resulted in noncritical slowing. As one expects, closer to the critical point, reaching the median branch requires relatively smaller values of $A_{S}$. Conversely, as the bias point is moved away from the critical point larger values of $A_{S}$ are needed to overcome critical slowing. In the latter regime, when the total input level during the switch is close to that corresponding to the critical point one expects a slower response of the system. This effect can be clearly seen in curve 1 , where the gradient increases with a more parabolic dependence on $A_{S}$ for small switch amplitudes.

One generally expects a trade-off to exist between switch power and system bandwidth. Here the lack of any visible saturation effects as $A_{S}$ increases seems to imply that lower switching times may be prescribed. This conjecture has been tested down to $T=1$, whereby solitary wave pixels were addressed in a single-cavity transit. ${ }^{6}$ We conclude that response for short, high-amplitude pulses is, to a good approximation, linear in pulse area even in our dispersive system and with transverse effects.

\section{RELAXATION AFTER ADDRESS PULSES}

To investigate the role of switch amplitude in determining the dynamics of a solitary pixel after an address pulse, we denote the output solitary amplitude $y(t)$, where $t$ is a discrete variable in units of cavity transit time. For any one bias point $A$ that implies bistability we define $\left(y_{1}, y_{u}, y_{2}\right)$ as the values of $y$ corresponding to the lower-, middle-, and upper-branch solutions, respectively. In studies of noncritical slowing it is common to define a lethargy time. For this purpose we assign the variable $\tau^{\prime}$ to the time taken for the metastable trajectory to cross the halfway point between $y_{u}$ and either $y_{1}$ or $y_{2}$. To distinguish upswitching or downswitching, we clarify the dynamics by defining a new variable $\tau= \pm \tau^{\prime}$, respectively. In Fig. 6a the logarithmic dependency of this lethargy time on the 

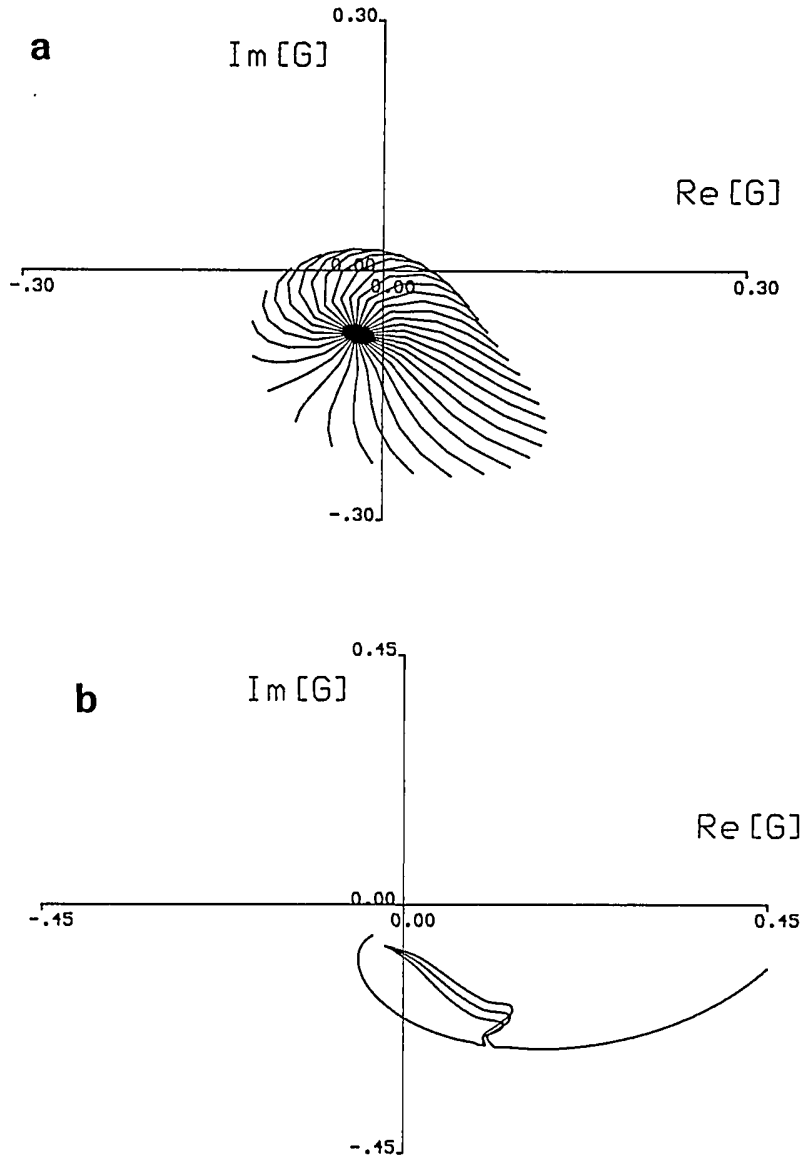

Fig. 7. Evolution of the output field in the complex plane $(A=0.12)$. a, Developments during a range of switch phases, $\psi \in[0,2 \pi)$. b, Trajectories during and after switching when $\psi=0, \pi / 16, \pi / 8$.

deviation of switch amplitude from its critical value $A_{S}{ }^{*}$ is investigated for three widely separated bias points. We determined the values for $A_{S} *$ previously from an intensive sequence of interpolative simulations for each $A$. The data show a good logarithmic dependency on $\left|A_{S}-A_{S}{ }^{*}\right|$ and equal gradients for the two signs of $\tau$ for $A=0.12$.

When $A_{S}$ departs sufficiently from $A_{S}{ }^{*}$, then our definition of lethargy time fails. The constraint that defines a meaningful lethargy time requires $\Delta$ " $-\infty$ as the critical points are approached, and, indeed, curve 1 shows a deviation from the scaling law when $\Delta$ is relatively large. One expects critical slowing to dominate the dynamics close to the critical points. Unfortunately, the precision and length of computations required for this transition region to be mapped out fully renders a study of this aspect outside the scope of this work.

In Fig. 6b, data for intermediate bias points are given, reaffirming the logarithmic dependency and equal gradients for upswitching and downswitching. Variation of gradients across the bias range is wide and varies approximately as $1 /\left(y_{2}-y_{u}\right)$, though we find that other, more symmetric, dependencies such as $\left(y_{u}-y_{1}\right) /\left(y_{2}-y_{u}\right)$ permit data fits that are just as good. ${ }^{20,26}$ One implication from these gradients is that the required resolution $\left|A_{S}-A_{S}{ }^{*}\right|$ for a specific lethargy becomes greatly relaxed as we approach the downswitching bias point. This is related to a decrease in the eigenvalue corresponding to $W^{u}$ as $A$ tends to the critical downswitch value.

\section{PHASE-ENCODED ADDRESS}

\section{A. Plane-Wave Limit}

We may generalize our input field by incorporating a definite phase $\psi$. Considering first the plane-wave limit, we write $g=|g| \exp (i \theta)$ and $a=|a| \exp (i \psi)$ and obtain, under stationary conditions,

$$
\begin{aligned}
|a|^{2} & =|g|^{2}\left[1+R^{2}-2 R \cos (\Gamma)\right], \\
\tan (\theta) & =\frac{\sin (\psi)+R \sin (\Gamma-\psi)}{\cos (\psi)-R \cos (\Gamma-\psi)}
\end{aligned}
$$

Thus the magnitude and, indeed, the existence of the fixed points depend only on the amplitude of the driving field. Finite $\psi$ causes only a rotation of the plane. A sudden change in address phase, say $a=|a|$ " $|a| \exp (i \psi)$, is thus equivalent to changing only the initial condition. Alternatively, we can envisage a fixed initial condition and a rotation of the stable manifold to cause a switch. Early studies of transient phenomena that are due to rapidly changing driving fields in dispersive optical bistability appreciated the complexity of motion in the phase plane. ${ }^{27,28}$

Figure 3 shows that switching purely by a sudden change in input phase is possible. To see this, one constructs circles around the origin whose radii are given by the magnitudes of the position vectors of the stable fixed points. The bands of parameter space where such switching is possible are delimited by the tangency of these circles with $W^{S}$.

\section{B. Solitary Wave Pixels}

We now permit our switch amplitude to become complex in the full transverse mapping, $A_{S}$ " $A_{S} \exp (i \psi)$, and explore how a combined change in both the amplitude and the phase of the local input field affects the on and off pixel sites. Immediately we can infer that during address there is a rotation and deformation of the appropriate manifolds with respect to the initial conditions.

At each bias point we have performed a range of switching simulations, each uniformly covering $\psi \in[0,2 \pi)$. Using the $(\psi=0)$ critical switch amplitude $A_{S}{ }^{*}$ permits discrimination between address phases through the noncritical slowing phenomenon. In this context we find that $\psi=0$ is not necessarily optimal, as a slightly positive value may cause switching. During address with $\psi \simeq \pi$ the phase evolutions show apparently anomalous jumps. Generally, by examining only the output phase during such numerical experiments one can also clearly see the noncritical slowing effect. However, monitoring of just the phase or intensity often shows curious relaxations and sudden jumps for a wide range of parameters. Address constitutes an energy injection that is not necessarily of the correct amplitude or phase for the target fixed point, and associated effects are more pronounced when large injections are considered. Relaxations and apparently anomalous jumps in amplitude or phase may be easily interpreted when the complex nature of the field is taken into account.

In Fig. 7a evolution of the solitary amplitude for a set of address pulses, for which $\left|A_{S}\right|=A_{S}{ }^{*}$, is shown in the complex field plane. In this representation a clear continuity between different address phases can be seen. The continuity within each trajectory is also clear, and we can simply interpret both the amplitude and the phase changes 


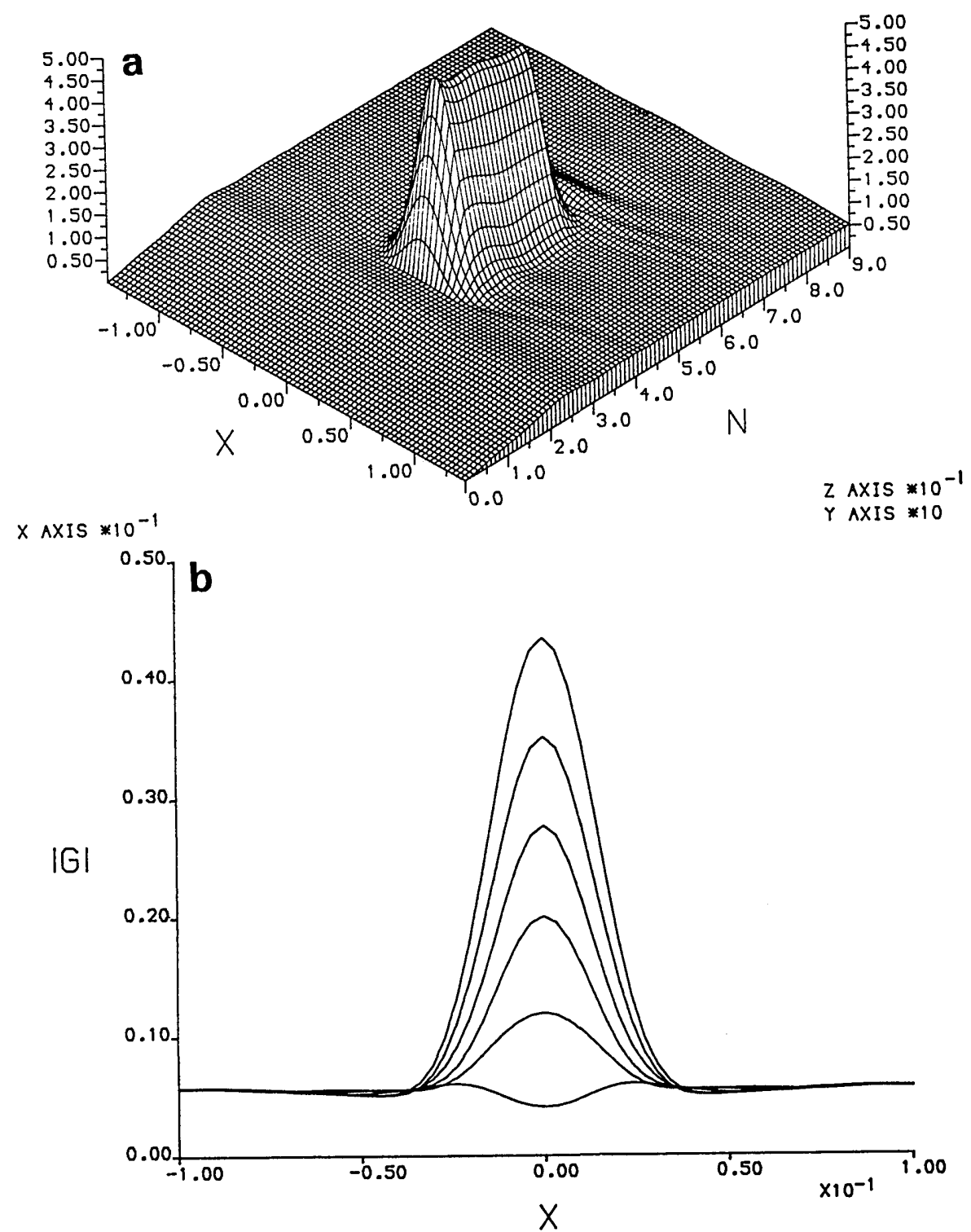

Fig. 8. Transverse profiles during a switch cycle of two address pulses, the second having an overall phase encoded $(A=0.095)$. a, Isometric plot; $N$ is in multiples of 10 , while $|G|$ is in units of 0.1 . b, Overlay of transverse profiles during solitary pixel reset.

that occur. Figure $7 \mathrm{~b}$ shows trajectories both during and after the switch for $\psi=n \pi / 16$ (where $n=0,1,2$ ). The effect of address phase is to separate these three solutions and prepare the system, after address, on either side and close to $W^{s}$. Consistent with the plane-wave dynamics, the trajectories then move approximately tangent to $W^{s}$ until $W^{u}$ is close. After this the $\psi=0.0$ solution remains near $M$ (noncritical slowing), while the other solutions depart toward their respective fixed points. The latter two trajectories are clearly indicative of a single curve, that of $W^{u}$. The phase of the field is seen to undergo rapid changes when a trajectory passes close to the origin. In Fig. 3 the intracavity plane-wave field was plotted, but here output solitary amplitude is shown. The former requires, at least, a rotation and dilation in the phase plane before quantitative correspondence can be established. A fuller examination of the dynamics reveals the stable fixed points to be spiral nodes, the degree of development of each spiral depending on relative distance between the bias point and each critical point.

For a wide range of bias points we have performed full clock cycles on the solitary pixels in which the pixels are first switched on and then off. For this operation it is possible to keep the amplitude of the address pulse fixed and simply vary $\psi$ to change its role. Choosing an amplitude for a $\psi=0$ switch-on pulse to give fast clean switching and a bias point close to the lower limit of the bistable range $(A=0.095)$, we find $\psi \simeq 2.75$ optimal for the reset pulse. This value leads to a smooth and fast annihilation of the solitary pixel. When $\psi \simeq \pi$ is implemented, the trajectory drawn out by the solitary amplitude executes a relatively large spiral around the lower-branch point before coming to rest. At the upper end of the bias range, shrinking of the $W^{s}$ lobe places greater constraints on the 


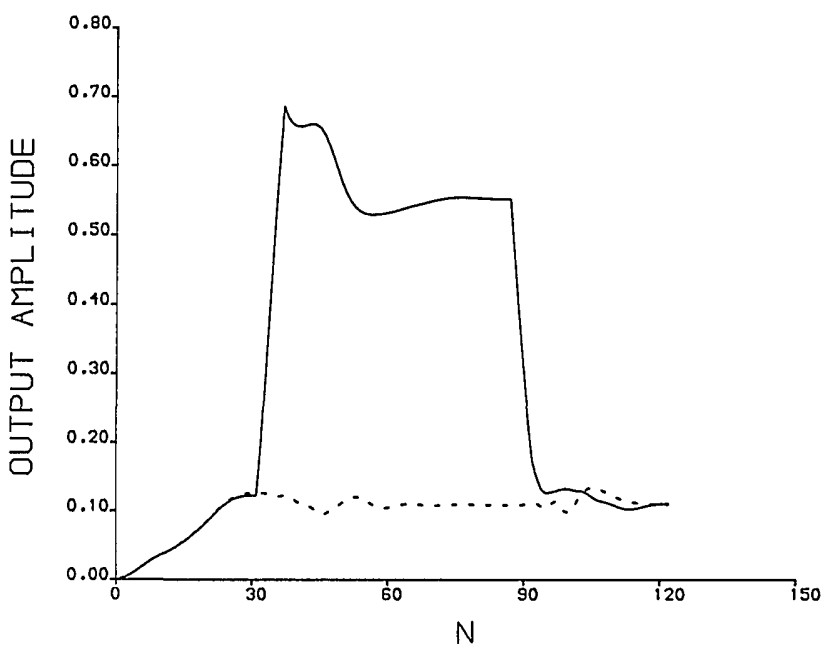

Fig. 9. Two solitary pixels, each adjacent to the central pixel site, are switched on and then off by use of address beams with $A_{S}=0.3166$ and $T=6$. Switch-on is performed with a real address pulse $(\psi=0)$, whereas the reset pulse has $\psi=3.53$. Shown are the peak amplitudes at beam center (dashed curve) and at one of the switched sites (bold curve).

value of $\psi$ that can effect a reset. However, even here, effective phases for the switch-off pulse still lie within the broad band that can be used when $A=0.095$.

\section{DYNAMICS OF THE TRANSVERSE PROFILE}

Until this point we have displayed only the dynamics of the solitary amplitude. We now show some of the accompanying transverse profiles during phase-encoded address. Figure 8a shows a complete switch cycle when the reset pulse has $\psi=2.75$. Inspection of just the solitary amplitude led to the deduction that this phase would be optimal. Mainly constructive interference results when $\psi=0.0$ and $\pi / 2$, while a rapid destruction of the whole pixel can be seen to occur here and also results from $\psi \approx \pi$. In this last case the spiraling of the solitary trajectory leads to on-axis oscillatory motion, which seems to drive a diffractive radiation spillage out of the pixel slot and across the beam. The dynamics of the solitary amplitude is thus a good indicator not only of switching speed but also of how cleanly the pixel is switched off. In Fig. 8b, transverse profiles during pixel reset with $\psi=2.75$ are shown and display a spectacularly clean switch-off. Here the solitary amplitude is switched to a point close to the lowerbranch node and subsequently remains in its vicinity.

To extend transverse considerations further, we demonstrate the use of a background beam with $A=0.1425$ on which a ...0010100 ... pattern is destructively reset to $\ldots 0000000 \ldots$. Here we are working at the top of the utilizable bias range, and, since we are also resetting two pixels adjacent to the central site, one expects a high likelihood that resulting radiation may activate switching at the central slot through trapping of diffractive waves. ${ }^{26}$ Many calculations have been performed at optimal $\psi$ for each value of $A$, and no such cross switching has been observed. The quoted exmple of a host beam close to the switchup point is shown in Fig. 9. Here the output amplitude of one of the switched pixels is plotted (solid curve) along with the field at the center of the beam (dashed curve). The only effect at the central (unaddressed) pixel site that is due to these large address pulses (at neighboring sites) is a small perturbation of the field. Notice also, in both the upswitching and downswitching processes, how close the system response is to linear. This smooth and controllable switching pattern can be compared with the more exponentially decaying (temporal) profiles that arise when beam interrupt is implemented for pixel reset.

\section{CONCLUSIONS}

Dynamical scalings and phased-encoded address beams in spatial solitary wave switching have been considered. The effect of noncritical slowing is found to be fundamental to transient dynamics, playing a major role in both upswitching and downswitching times of solitary pixels in a system in which, implicitly, transverse effects are central. Static and dynamic separatrices for the switching process have been determined. For transient dynamics after the address of a pixel we found a logarithmic scaling in terms of $A_{S}{ }^{*}$ (which was predicted in plane-wave models) to be valid over nearly the whole bias range.

In the most relevant situation of short, high-amplitude address pulses, we found system response not only temporally linear but also linear in pulse area (consistent across the bias range). This generalization of the (plane-wave) pulse area theorem to a domain with significant transverse effects is both remarkable and useful. The relation was used, though not stated, in a previous paper ${ }^{6}$ to determine parameters such that solitary wave switching in the duration of a single-cavity transit could be attained. Manifolds and dynamic flow vectors of the plane-wave map have been calculated and have been used to explain switching features of the full transverse switching scheme. Smoothness of the transient transverse structures and the constrained motion of the pixel amplitude in the phase plane indicate large-scale simplication of the possible complex dynamics.

We determined bands of address phase for solitary wave pixel destruction for a situation in which on and off pulses have equal magnitude. The allowable band of phase shrinks at higher bias levels but still permits single values to be applicable to nonuniform arrays. Cross-talk-induced switching has been tested for, in likely configurations, and results show that this unwanted switching is avoidable.

While for many materials finite medium response time may limit switching speed and introduce new effects, we have focused here on a purely dispersive system and the accommodation of transverse effects. In earlier studies ${ }^{5}$ it was proved that spatial solitary waves dominate the asymptotic stationary output patterns that arise from a transverse modulational instability of the circulating light beam. Here we have presented evidence that strongly suggests that the same spatially coherent attractors dominate the switching dynamics of the system. The case for reduction of the spatiotemporal complexity through particlelike solitary wave characteristics has been supported by demonstration of noncritical slowing, generalization of the logarithmic scaling law, demonstration of the validity of the pulse area theorem and the continuity into the domain of critical slowing, a direct comparison of the complex phase dynamics in the plane-wave limit and those of the complex solitary amplitudes, and finally an application of these results in which the spatial structures 
can be controlled to the extent that they are individually switched on or off according to the phase of an external clock pulse.

\section{ACKNOWLEDGMENTS}

This study was supported in part by a Science and Engineering Research Council (SERC) Studentship and in part by SERC grants GR/F 76502 and GR/G 12665. We acknowledge constructive comments made by one of the reviewers.

*Present address, Optique Non-Linèaire Théorique, Université Libre de Bruxelles, Campus Plaine CP 231, 1050 Bruxelles, Belgium.

\section{REFERENCES}

1. N. B. Abraham and W. J. Firth, "Overview of transverse effects in nonlinear-optical systems," J. Opt. Soc. Am. B 7, 951 (1990).

2. R. De La Fuente, A. Barthelemy, and C. Froehly, "Spatialsoliton-induced.guided waves in a homogeneous nonlinear Kerr medium," Opt. Lett. 16, 793 (1991).

3. S. Maneuf and F. Reynaud, "Quasi-steady state self-trapping of first, second and third order subnanosecond soliton beams," Opt. Commun. 66, 325 (1988).

4. J. S. Aitchison, A. M. Weiner, Y. Silberberg, M. K. Oliver, J. L. Jackel, D. E, Leaird, E. M. Vogel, and P. W. E. Smith, "Observation of spatial optical solitons in a nonlinear glass waveguide," Opt. Lett. 15, 471 (1990).

5. H. Adachihara, D. W. McLaughlin, J. V. Moloney, and A. C Newell, "Solitary waves as fixed points of infinitedimensional maps for an optical bistable ring cavity: analysis," J. Math. Phys. 29, 63 (1988).

6. G. S. McDonald and W. J. Firth, "All-optical switching in a nonlinear resonator," J. Mod. Opt. 37, 613 (1990).

7. G. S. McDonald and W. J. Firth, "Spatial solitary-wave optical memory," J. Opt. Soc. Am. B 7, 1328 (1990).

8. H. A. Haus and A. Mecozzi, "Long-term storage of a bit stream of solitons," Opt. Lett. 17, 1500 (1992).

9. N. A. Whitaker, Jr. and H. Avramopoulos, "Addressable fiber loop memory," presented at the International Quantum Electronics Conference, Vienna, Austria, June 14-19, 1992.
10. R. Bonifacio and L. Lugiato, "Cooperative effects and bistability for resonance fluorescence," Opt. Commun. 19, 172 (1976).

11. R. Bonifacio and P. Meystre, "Critical slowing down in optical bistability," Opt. Commun. 29, 131 (1979).

12. F. A. Hopf and P. Meystre, "Numerical studies of the switching of a bistable optical memory," Opt. Commun. 29, 235 (1979).

13. T. Erneux and P. Mandel, "Temporal aspects of absorptive optical bistability," Phys. Rev. A 28, 896 (1983).

14. P. Mandel, "Switching pulses for an optical transistor," Opt. Commun. 54, 374 (1985).

15. G. Grynberg and S. Cribier, "Critical exponents in dispersive optical bistability," J. Phys. Lett. 44, 449 (1983).

16. E. Abraham and C. Rae, "Cross talk in nonlinear interference filters: loop narrowing and critical slowing down," J. Opt. Soc. Am. B 4, 490 (1987).

17. P. Mandel, "Scaling properties of switching pulses," Opt. Commun. 55, 293 (1985)

18. B. Segard, J. Zemmouri, and B. Macke, "Switching delays in optical bistability: an experimental study," Opt. Commun. 60, 323 (1986).

19. J. Y. Bigot, A. Daunois, and P. Mandel, "Slowing down far from the limit points in optical bistability," Phys. Lett. A 123, 123 (1987).

20. B. Segard and B. Macke, "Non critical slowing down in optical bistability," J. Phys. 49, 115 (1988).

21. B. Segard, J. Zemmouri, and B. Macke, "Noncritical slowing down in optical bistability," Opt. Commun. 63, 339 (1987).

22. J. Y. Bigot, A. Daunois, and J. B. Grun, "Switching dynamics of thermally induced optical bistability: theoretical analysis," Phys. Rev. A 35, 3810 (1987).

23. B. Honerlage and J. B. Grun, "Switching dynamics of dispersive optical bistable devices, using three-level systems as active media," Europhys. Lett. 3, 681 (1987).

24. F. Mitschke, C. Boden, W. Lange, and P. Mandel, "Exploring the dynamics of the unstable branch of bistable systems," Opt. Commun. 71, 385 (1989).

25. S. M. Hammel, C. K. R. T. Jones, and J. V. Moloney, "Global dynamical behavior of the optical field in a ring cavity," J. Opt. Soc. Am. B 2, 552 (1985).

26. G. S. McDonald, "Spatial solitary wave optical memory," $\mathrm{Ph} . \mathrm{D}$. dissertation (Strathclyde University, Glasgow, Scotland, 1989).

27. F. A. Hopf, P. Meystre, F. D. Drummond, and D. F. Walls, "Anomalous switching in dispersive optical bistability," Opt. Commun. 31, 245 (1979).

28. F. A. Hopf and P. Meystre, "Phase-switching of a dispersive non-linear interferometer," Opt. Commun. 33, 225 (1980). 